\title{
Molecular Epidemiology and Evolution of Equine Arteritis Virus
}

UDENI B. R. BALASURIYA, JODI F. HEDGES, AND N. JAMES MACLACHLAN

Bernard and Gloria Salick Equine Viral Disease Laboratory, Department of Pathology,

Microbiology and Immunology, School of Veterinary Medicine, One Shields Avenue, University of California, Davis, CA 95616

\section{INTRODUCTION}

EVA is an infectious disease of equids that occurs in many parts of the world and is caused by EAV (Timoney and McCollum, 1993; Glaser et al., 1997). Most EAV infections are subclinical but occasional outbreaks of disease occur that are characterised by any combination of influenza-like illness in adult horses, abortion in pregnant mares, and interstitial pneumonia in very young foals. Up to $60 \%$ of stallions infected with EAV become persistently infected carriers and shed virus continuously in semen. Persistence of EAV in the male reproductive tract is testosterone-dependent, and persistently infected stallions function as a natural reservoir that can disseminate virus to susceptible mares at breeding (Timoney and McCollum, 1993).

The two principal modes of EAV transmission are horizontal transmission by aerosolization of infectious respiratory tract secretions from acutely infected horses and venereal transmission during natural or artificial insemination with infective semen from persistently infected stallions (Timoney and McCollum, 1993; Glaser et al., 1997). EAV also can be transmitted through indirect contact with formites or personnel. Congenital infection results from transplacental transmission (vertical transmission) of virus when pregnant mares are infected late in gestation.

There is only one serotype of EAV and all field isolates evaluated 
thus far are neutralised by polyclonal antiserum raised against the VBS53 strain, however, geographically and temporally distinct strains of EAV differ in the severity of the clinical disease they induce and in their abortigenic potential (Timoney and McCollum, 1993). There is considerable genetic variation amongst field strains of EAV (Balasuriya, 1995, 1998 and 1999; Hedges et al., 1996 and 1999; Patton et al., 1998; Stadejek et al., 1999; Chirnside et al., 1994; Archambault et al., 1997).

We have focused our recent studies on the molecular epidemiology and evolution of EAV to better characterise the role of the carrier stallion in the emergence of novel genotypic and phenotypic variants of the virus.

\section{MATERIALS AND METHODS}

Viral RNA directly isolated from nasal swabs, mononuclear cells and from the semen of two stallions that were experimentally inoculated with the virus derived from the infectious cDNA clone of EAV was used to characterise the genetic heterogeneity of EAV during transient infection of horses (Balasuriya et al., 1999). To study viral heterogeneity during acute infections, viral RNA was isolated directly from homogenates of placenta from one aborted foetus and six congenitally infected foals, and from tissues of two foals that died during an outbreak of EVA (Balasuriya et al., 1999). Viral RNA was also isolated from the semen of carrier stallions naturally infected with EAV (Balasuriya et al., 1999; Hedges et al., 1999). ORFs 2-7 and flanking portions of the EAV genome (2923 bp) were RT-PCR amplified by RT-PCR using Superscript II and $P f u$ Turbo DNA polymerase, as previously described (Balasuriya et al., 1999; Hedges et al., 1999). Individual cDNA amplicons of ORF5 within each RT-PCR sample were cloned into a vector and 14-20 clones were compared to determine the quasispecies heterogeneity of each sample.

\section{RESULTS}

The master sequence of the virus present in the nasal swabs, buffy coat cells and semen collected from one stallion after experimental infection with the recombinant virus was identical to that of the cloned EAV030H virus used to infect the horses. There was little heterogeneity $(2 / 20)$ in the clones derived from the virus used to inoculate the horses or in the mononuclear cells collected at 8 DPI (2/20 clones), whereas there were substitutions in 15/20 clones of ORF5 of EAV in the mononuclear cells collected from 14 days post inoculation (DPI) and in all 20 clones from the mononuclear cells collected from the second stallion at $10 \mathrm{DPI}$. The data 
indicate that although the master sequence of the recombinant virus was conserved during infection of the two stallions, the genetic heterogeneity of the virus population increased in the course of acute transient infection.

We also investigated an extensive outbreak of EVA on a Warmblood breeding farm to characterize the molecular epidemiology of EAV infection and to determine the genetic heterogeneity that was generated during this outbreak. Sequence and phylogenetic analysis of ORFs 2-7 of the master sequences of viruses that were present in foal tissues and sequential semen samples from four persistently infected stallions on the farm demonstrated that a virus in the semen of one carrier stallion initiated the outbreak. The virus in the lung of the first foal that died during the outbreak was very similar ( $99.5 \%$ identity) to virus in a semen sample that was collected from one carrier stallion 4 days prior to the outbreak. There were no obvious sequence differences between viruses present in the tissues of the aborted foal, foals that developed pneumonia or systemic illness, and in the placentas of foals that exhibited no clinical evidence of EAV infection ( $>99.9 \%$ identity) during the outbreak. In contrast, viral sequences derived from the semen of the two stallions that became persistently infected during the outbreak differed by 44 nucleotides $(98.4 \%$ identity) after 12 months of persistent infection, indicating that each virus evolved independently during the course of persistent infection. The ORF5 sequences of clones derived from the RT-PCR products directly amplified from the semen of three carrier stallions (the stallion that initiated the outbreak and two stallions that became persistently infected following the outbreak) and tissues from three foals, were compared to further characterize the genetic heterogeneity of the EAV quasispecies during horizontal and vertical transmission. A total of 78 clones that included the entire ORF5 sequence were analyzed, including 14 to 20 clones from each semen sample and 28 clones from the various foal tissues. The clones from the semen of two stallions that became persistently infected following the outbreak showed increased genetic heterogeneity during the course of persistent infection. In contrast, the clones from the outbreak were homogeneous.

To characterize the genetic heterogeneity of EAV during persistent infection, detailed sequence analysis of the structural protein genes (ORFs 2-7) was performed with viral RNA purified directly from semen collected sequentially over a 10 -year period from two Thoroughbred carrier stallions that were infected during an EVA outbreak in Kentucky in 1984 (Hedges et al., 1999). Sequence analysis of ORFs 2-7 clearly indicated that the RNA in the semen of the two stallions was distinct from year to year. The master sequence of the virus population shed in the semen of individual stallions varied by approximately $1 \%$ per year. ORFs 2-7 of major EAV variants (master sequences) present in the semen of the two carrier stallions evolved independently of each other. Overall, ORFs 3 and 5 evolved most rapidly, 
ORFs 2 and 4 moderately, and ORFs 6 and 7 were substantially more conserved during persistent EAV infection.

Variation occurred in the $G_{L}$ protein during persistent infection of stallions primarily within a specific section of the Vl region that expresses three of the major virus neutralization sites. The neutralization phenotype of selected sequential EAV isolates from the semen of the two carrier stallions was evaluated by neutralization assay with a large panel of monoclonal antibodies and polyclonal equine antisera. There were significant differences in the neutralization phenotype of some of the sequential isolates. The data clearly indicate that selective pressures exerted during the course of persistent EAV infection of stallions significantly influence the evolution of specific regions of the $G_{L}$ protein as variants with altered neutralization phenotypes emerge.

To further characterize the EAV quasispecies present during persistent infection of stallions, ORF 5 was RT-PCR amplified and cloned from viral RNA isolated directly from the semen collected in 1984 and 1994 from one stallion (17 and 16 clones respectively). Most ORF5 clones were distinct, indicating that EAV exists as a mixture of related genomes. The clones from semen collected in 1994 were more variable than the clones derived from semen collected from the same stallion 10 years earlier. Sequence and phylogenetic analysis of the various clones clearly showed that the heterogeneity of the quasispecies increased during the course of long-term persistent EAV infection of the stallion.

\section{DISCUSSION}

In our studies of the molecular evolution of EAV, we have shown that virus derived from an infectious cDNA clone is genetically stable during acute, transient infection. The genetic stability of viruses that circulated during an outbreak of EVA contrasts markedly with the heterogeneous virus populations present in the semen of persistently infected stallions. Selective pressures exerted during the course of persistent infection of the reproductive tract of the carrier stallion clearly can be responsible for genotypic divergence and emergence of phenotypically novel EAV variants, and likely compensate for the relatively limited diversity of EAV that is generated during EVA outbreaks. Novel viral strains can emerge that are capable of initiating outbreaks of EVA. Thus, the persistently infected carrier stallions acts as a reservoir that harbors the EAV between breeding seasons, during which viral genetic diversity increases. 


\section{ACKNOWLEDGMENTS}

These studies were supported by the Grayson-Jockey Club Research Foundation Inc., the Center for Equine Health at the University of California-Davis, the Bernard and Gloria Salick Endowment, and USDA Competitive Grant 97-35204-4736. We would like to thank Dr. Eric J. Snijder for providing the infectious cDNA clone of EAV and Drs. William $\mathrm{H}$. McCollum and Peter J. Timoney for providing various field stains of the virus.

\section{REFERENCES}

Archambault, D., Laganiere, G., Carman, S., and St-Laurent, G.(1997). Comparison of nucleic and amino acid sequences and phylogenetic analysis of open reading frames 3 and 4 of various equine arteritis virus isolates. Vet.Res. 28, 505-516.

Balasuriya, U.B.R., Evermann, J.F., Hedges, J.F., McKeirnan, A.J., Mitten, J.Q., Beyer, J.C., McCollum, W.H., Timoney, P.J., and MacLachlan, N.J.(1998). Serologic and molecular characterization of an abortigenic strain of equine arteritis virus derived from infective frozen semen and an aborted equine fetus. J.Am.Vet.Med.Assoc. 213, 1586-1589.

Balasuriya, U.B.R., Hedges, J.F., Timoney, P.J., McCollum, W.H., and MacLachlan, N.J.(1999). Genetic stability of equine arteritis virus during horizontal and vertical transmission in an outbreak of equine viral arteritis. J.Gen.Virol. 80, 1949-1958.

Balasuriya, U.B.R., Snijder, E.J., van Dinten, L.C., Heidner, H.W., Wilson, W.D., Hedges, J.F., Hullinger, P.J., and MacLachlan, N.J.(1999). Equine arteritis virus derived from an infectious cDNA clone is attenuated and genetically stable in infected stallions. Virology 260, 201-208.

Balasuriya, U.B.R., Timoney, P.J., McCollum, W.H., and MacLachlan, N.J.(1995).

Phylogenetic analysis of open reading frame 5 of field isolates of equine arteritis virus and identification of conserved and nonconserved regions in the $G_{L}$ envelope glycoprotein. Virology 214, 690-697.

Chirnside, E.D., Wearing, C.M., Binns, M.M., and Mumford, J.A.(1994). Comparison of M and $\mathrm{N}$ gene sequences distinguishes variation amongst equine arteritis virus isolates. J.Gen.Virol. 75, 1491-1497.

Hedges, J.F., Balasuriya, U.B.R., Timoney, P.J., McCollum, W.H., and MacLachlan, N.J.(1996). Genetic variation in open reading frame 2 of field isolates and laboratory strains of equine arteritis virus. Virus Res. 42, 41-52.

Hedges, J.F., Balasuriya, U.B.R., Timoney, P.J., McCollum, W.H., and MacLachlan, N.J.(1999). Genetic divergence with emergence of phenotypic variants of equine arteritis virus during persistent infection of stallions. J.Virol. 73, 3672-3681.

McCollum, W.H., Prickett, M.E., and Bryans, J.T.(1971). Temporal distribution of equine arteritis virus in respiratory mucosa, tissues and body fluids of horses infected by inhalation. Res.Vet.Sci. 12, 459-464.

Patton, J.F., Balasuriya, U.B.R., Hedges, J.F., Schweidler, T.M., Hullinger, P.J., and MacLachlan, N.J.(1998). Phylogenetic characterization of a highly attenuated strain of equine arteritis virus from the semen of a persistently infected standardbred stallion. Arch.Virol. 144, 817-827. 
Stadejek, T., Bjorklund, H., Ros Bascunana, C., Ciabatti, I.M., Scicluna, M.T., Amaddeo, D., McCollum, W.H., Autorino, G.L., Timoney, P.J., Paton, D.J., Klingeborn, B., and Belak, S.(1999). Genetic diversity of equine arteritis virus. J.Gen.Virol. 80, 691-699.

Timoney, P.J. and McCollum, W.H.(1993). Equine viral arteritis. Vet.Clin.N.Am., Eq.Pract. 9, 295-309. 